\title{
Design of Interval Type 2 Fuzzy Fault-Tolerant Controller for a Non-Minimum Phase System: Application to quadruple conical tank system
}

\author{
Himanshukumar R. Patel ${ }^{a}$ and Vipul A. Shah ${ }^{b}$ \\ ${ }^{a}$ Instrumentation and Control Engineering Department, Faculty of Technology, \\ Dharmsinh Desai University, Nadiad-387001, Gujarat, India, e-mail: himanshupatel.ic@ddu.ac.in \\ ${ }^{b}$ Instrumentation and Control Engineering Department, Faculty of Technology, \\ Dharmsinh Desai University, Nadiad-387001, Gujarat, India, e-mail: vashah.ic@ddu.ac.in
}

\begin{abstract}
Quadruple Conical Tank System (QCTS) is benchmark a laboratory setup for testing of various linear and nonlinear control algorithms for the multivariate control system. The multiple process configuration for multivariate input-output can be obtained from quadruple conical tank system. This article is concerned with designing a novel FaultTolerant Control (FTC) using Interval Type 2 Fuzzy Control (IT2FLC) subject to actuator and system component (Leak) faults. Additionally, paper explores the pen loop response with given operating points of quadruple conical tank system linearized model for non-minimum phase configuration obtained from MATLAB. The proposed control design method is simulated on prototype model of quadruple conical tank process for onminimum phase configuration. The performance of the proposed controller is studied through extensive simulations with regulatory and servo responses. Through comparative studies of proposed controller with other model based $\mathcal{H}_{\infty}$, LQR, 2 DoF Decentralized PI Controller and model free Type 1 Fuzzy Logic controller (T1FLC) responses in MATLAB simulation environment, the effect of interaction, coupling, time variations and change in dynamic behaviour of QCTS confirmed.
\end{abstract}

Keywords: Actuator fault, Fault-tolerant control, Quadruple Conical Tank System, non-minimum phase system, system component fault, interval type 2 fuzzy control.

\section{Introduction}

The majority number of chemical processes are multivariable in nature, exhibiting some strong couplings and occasionally a non-minimum phase character that makes the control design problem a challenging task [4], [25], [37]. In general, for such chemical processes, the objective of a control system is to maintain several controlled variables at independent set points. Despite the coupling problems associated with multivariable systems, a non-minimum phase system is even more difficult to control. None of the techniques that are based upon model inversion can be used since such an inversion leads to an unstable closed loop system. Multivariable controllers have been previously designed for such systems. However, centralized controller design for multiple-input-multiple-output (MIMO) systems is associated with possible problems concerning the complex computations, maintenance due to the size and a high risk of failure even though it provides better performance. Simplified algorithms are generally preferred as an alternative solution. In contrast to the centralised multivariable control, decentralised control is widely preferred in practice and industrial applications especially because of its main advantage that allows for an easy implementation and tuning, if a sufficient number of sensors and actuators exist. It is also highly reliable and flexible. If properly tuned, it can lead to excellent closed loop results. Although, many other alternatives solutions ware provided for controlling MIMO non-minimum phase system like $\mathcal{H}_{\infty}$, LQR, 2 DoF Decentralized PI Controller and model free Type 1 Fuzzy Logic controller (T1FLC) in [3], [32], [33] and [34].

The quadruple tank process is the case study considered in this paper. These particular processes have been the focus of numerous papers, since they exhibit elegantly complex dynamics of interest in both control and research education [15], [25], [33] and [38]. They have been widely used in chemical engineering laboratories to illustrate the performance limitations 
for multivariable systems due to strong interactions, right-half plane transmission zeros and model uncertainties, the challenges and some solution discussed in [3], [10], [15], [38] and [34]. In [8], [10] and [11] authors are describe the effects of interaction in the system response and overcome this limitation with conventional PID controller structure using decentralized mode. In [15] authors design robust controller for quadruple level system using sliding-mode controller with fuzzy logic.Author in [38] designed decentralize controller and overcome the limitation of interaction, coupling in MIMO quadruple tank level process.In [37] nonlinear feedback controller is design for multivariable non-minimum-phase processes.

A passive Fault-tolerant control was design using conventional and artificial intelligence based techniques in [18, 20, 21, 22, 24, 26, 27, 28, 29, 34] and [39] for single input single output (SISO) and multi input multi output (MIMO) cylindrical tank level process subject to actuator, sensor, and system component (Leak) faults. However, in [1], [5], [19], [21], [23], [26] and [30, 31] instead of cylindrical tank authors taking conical shape tank, which is having highly nonlinear dynamics due to its shape, also in [1] and [5] author has design controller for quadruple conical tank system with nonminimum and minimum phase configuration. In [21], author design FTC using neural network for SISO Two-conical tank system subject to all possible fault in the system, but the structure of the controller is combination of both conventional PID controller and neural network.

IT2 FLCs are also appreciated and latest trends in control applications because they also provide a nonlinear control, but in addition provide the uncertainty consideration in their model, and some examples of applications of IT2 FLCs are [2], [7], [17], [35] and [40]

The main motive to go with the Interval Type-2 Fuzzy Logic System (IT2FLS) approach is list out as follows:

- The main advantages of the IT2FLC are providing robustness, free-model design, and better performance than classic alternatives, and this is mainly thanks to its non-linear modeling abilities. In [12] Hagras presents the advantages of modeling the uncertainty in the FLCs and how T2 FLCs offers an improvement with respect to T1 FLCs, in [41] $\mathrm{Wu}$ provides an analysis of the differences between T1 and IT2 FLC and in [6] \& [35] provides a performance comparison for a particular complex applications.

- The IT2 FLC controller is more robust against the noise environment [9] and it is best suitable for the nonlinear higher order system.
- IT2 FLC will incorporate the model uncertainty with the help of Footprint of Uncertainty (FOU) in IT2 FS, the concept of this well define in [26] and $[31]$.

- As discussed in [41] two fundamental differences between IT2 and T1 FLCs is 1) Adaptiveness and 2) Novelty. As a result, an IT2 FLC can implement a complex control surface that cannot be achieved by a T1 FLC using the same rule base.

Based on the above motivation and some literature in recent years on IT2 FLC we proposed IT2FLC based Fault Tolerant control (FTC) system for non-minimum phase system against different faults and model incertitude.

To summarize, the main contributions of the article are enumerated below: While the existing methods are, in general, highly iterative in nature and complex to implement, IT2FLC based controller is non-iterative synthesis method and knowledge base controller which reduce computational burden. A comparative results are produce, and confirm with three integral errors. Also transient responses are compared to other methods with proposed method. The proposed method is tested for a quadruple-tank system with non-minimum phase setting. The superiority of the proposed control over the widely used decentralized PI and other centralized controllers (i.e T1FLC, LQR, $\mathcal{H}_{\infty}$ is studied through extensive simulations). The paper presents remarkable results for highly nonlinear MIMO QCTS system with two faults.

The paper is structured as follows. The first section contains the introduction of the multivariable system and related literature survey, including a conventional PID as well as a decoupling, fuzzy logic, LQR, $\mathcal{H}_{\infty}$ approach. Then, the prototype model of QCTS is demonstrated, for case study the non-minimum phase conical water tanks system is taken. The third section presents the FTC design using IT2FLC. The fourth section presents the simulation results of servo and regulatory performance, while the main conclusions are stated in the final section of the paper.

\section{Prototype System Overview}

As shown in figure-1, MIMO Quadruple Conical Tank System (QCTS) prototype setup consist of four interconnected conical tanks, two water pump, one storage tank, two three-way valve, four hydrostatic level sensors. Some recent research work presents prototype system of QCTS in [1] [5]. In order to introduce coupling effect between inputs and outputs, three-way valves are used. The valve positions are $\lambda_{1}$ and $\lambda_{2}$. These valve positions give the ratio in which the out- 
put from the pump is divided between the upper and lower tanks. Input flow rates through final control assembly (VFD and pump) are manipulating inputs and level of the lower two tank that is tank 1 and tank 2 are controlled variables. The outlet of both pumps are connected with three-way valves. Notation for input variable are $v 1$ and $v 2$, which represents input flow rate to the system, and $h_{1}$ and $h_{2}$ represent the height of lower two tanks. Based on the operating point of three-way interaction valve input flow for tank 3,4 and tank 1, 2 vary. Apart from that, each tank has outlet with manual 2 ways valve, which are mentioned as $V_{1}, V_{2}, V_{3}$, and $V_{4}$ respectively, and each manual 2 way valve having valve co-efficient $\gamma_{1}, \gamma_{2}, \gamma_{3}$, and $\gamma_{4}$ respectively . Furthermore, in QCTS prototype, two faults are taken one is actuator faults $\left(f_{a}\right)$ and second one is system component (leak) faults $\left(f_{\text {sys }}\right)$. Using Control System and simulation tool box of MATLAB, detailed comparative analysis of open loop and closed loop responses in simulation is carried out.

The operation of quadruple tank system can be comprehended in two phases namely minimum phase and non- minimum phase. Minimum Phase: When the liquid enter the lower tanks is less than that of upper tanks then the system starts operating in minimum phase. Non-Minimum phase: when fraction of liquid entering the upper tanks is less than that of lower tanks, then the system starts operating in nonminimum phase.

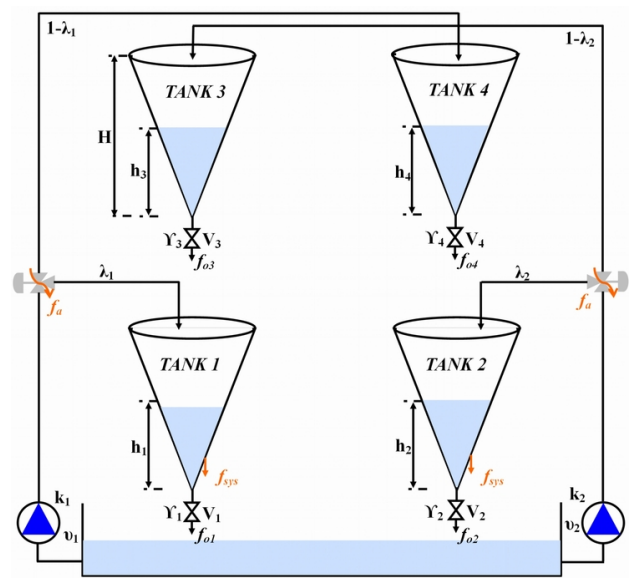

Figure 1: Schematic diagram of prototype QCTS.

\subsection{Mathematical Model of QCTS}

Quadruple tank system is very popular in academia. Based on newtons mass flow and Bernoullis law on mathematical model for QCTS system is obtained. After the linearization, the state space matrix A, B, $\mathrm{C}$, and $\mathrm{D}$ give transfer function matrix with the relation between inflow rate and height as the level of the tank. For the model of QCTS system input variable are $v 1$ and $v 2$ Inflow rates and the output variable is height or level of tank $h_{1}$ and $h_{2}$. To derive a mathematical model of QCTS system MATLAB platform is used. By using MATLAB Control System Toolbox; ordinary differential equations can be linearized with transfer function matrix. A complete linearized mathematical model is derived using control design and simulation tool box. MATLAB Control System Toolbox provides linearized transfer function matrix based on mass flow equation of QCTS system for a various operating point. In QCTS system two inputs and two outputs will generate $2 \times 2$ transfer function matrix with some dynamics like first order, second order and so on. Ordinary Differential Equation (ODE) for QCTS is as listed in equations 1 to 4 . Figure 1 shows the schematic diagram of QCTS with different parameters.

The notations used in figure- 2 are as below:

$K_{1}, K_{2}=$ pump gain $\left(\mathrm{cm}^{3}\right) / \mathrm{sec} /$ volt

$h_{1}, h_{2}, h_{3}, h_{4}=$ level of water or liquid in $\mathrm{cm}$

$v 1, v 2=$ voltage of pump (volt)

$\lambda_{1}, \lambda_{2}=$ inflow rate of pump1 and pump2 in $\mathrm{cm}^{3} / \mathrm{sec}$

$A_{1}, A_{2}, A_{3}, A_{4}=$ tank cross section area in $\mathrm{cm}^{2}$

$\mathrm{g}=$ gravitational force $=980.665 \mathrm{~cm} / \mathrm{s}^{2}$

$a_{1}, a_{2}, a_{3}, a_{4}=$ cross section area of outlet hole in $\mathrm{cm}^{2}$

$\gamma_{1}, \gamma_{2}, \gamma_{3}, \gamma_{4}=$ outlet valve ratio (co-efficient)

Now differential equations of QCTS are as listed below:

$$
\begin{gathered}
d h_{1} / d t=\frac{3}{A_{1}}\left\{\gamma_{3} a_{3} \sqrt{\left(2 g h_{3}\right)}+\lambda_{1} K_{1} v_{1}-\gamma_{1} a_{1} \sqrt{\left(2 g h_{1}\right)}\right\} \\
d h_{2} / d t=\frac{3}{A_{2}}\left\{\gamma_{4} a_{4} \sqrt{\left(2 g h_{4}\right)}+\lambda_{2} K_{2} v_{2}-\gamma_{2} a_{2} \sqrt{\left(2 g h_{2}\right)}\right\} \\
d h_{3} / d t=\frac{3}{A_{3}}\left\{\left(1-\lambda_{2}\right) K_{2} v_{2}-\gamma_{3} a_{3} \sqrt{\left(2 g h_{3}\right)}\right\} \\
d h_{4} / d t=\frac{3}{A_{4}}\left\{\left(1-\lambda_{1}\right) K_{1} v_{1}-\gamma_{4} a_{4} \sqrt{\left(2 g h_{4}\right)}\right\}
\end{gathered}
$$

The state space representation of QCTS is define by:

$$
\begin{aligned}
{\left[\begin{array}{l}
x_{1} \\
x_{2} \\
x_{3} \\
x_{4}
\end{array}\right]=} & {\left[\begin{array}{cccc}
\left(\frac{-1}{\tau_{1}}\right) & 0 & \left(\frac{1}{\tau_{1}^{\prime}}\right) & 0 \\
0 & \left(\frac{-1}{\tau_{2}}\right) & 0 & \left(\frac{-1}{\tau_{2}^{\prime}}\right) \\
0 & 0 & \left(\frac{1}{\tau_{3}}\right) & 0 \\
0 & 0 & 0 & \left(\frac{1}{\tau_{4}}\right)
\end{array}\right]\left[\begin{array}{l}
x_{1} \\
x_{2} \\
x_{3} \\
x_{4}
\end{array}\right]+} \\
& {\left[\begin{array}{cc}
\frac{3 \gamma_{1} k_{1}}{A_{1}} & 0 \\
0 & \frac{3 \gamma_{2} k_{2}}{A_{2}} \\
0 & \frac{\left(1-\gamma_{2}\right) k_{2}}{A_{2}} \\
\frac{\left(1-\gamma_{1}\right) k_{1}}{A_{2}} & 0
\end{array}\right]\left[\begin{array}{l}
u_{1} \\
u_{2}
\end{array}\right] }
\end{aligned}
$$


where;

$$
\begin{gathered}
\frac{1}{\tau_{1}}=\frac{3 \gamma_{1} a_{1} \sqrt{(2 g)}}{A_{1}\left(2 \sqrt{\left(h_{1}\right)}\right)}, \frac{1}{\tau_{1}^{\prime}}=\frac{\gamma_{3} a_{3} \sqrt{(2 g)}}{A_{1}\left(2 \sqrt{\left(h_{3}\right)}\right)} \\
\frac{1}{\tau_{2}}=\frac{3 \gamma_{2} a_{2} \sqrt{(2 g)}}{A_{2}\left(2 \sqrt{\left(h_{2}\right)}\right)}, \frac{1}{\tau_{2}^{\prime}}=\frac{3 \gamma_{4} a_{4} \sqrt{(2 g)}}{A_{4}\left(2 \sqrt{\left(h_{4}\right)}\right)} \\
\frac{1}{\tau_{3}}=\frac{3 \gamma_{3} a_{3} \sqrt{(2 g)}}{A_{3}\left(2 \sqrt{\left(h_{3}\right)}\right)}, \frac{1}{\tau_{4}}=\frac{3 \gamma_{4} a_{4} \sqrt{(2 g)}}{A_{4}\left(2 \sqrt{\left(h_{4}\right)}\right)} \\
{\left[\begin{array}{l}
y_{1} \\
y_{2}
\end{array}\right]=\left[\begin{array}{cccc}
K_{c} & 0 & 0 & 0 \\
0 & K_{c} & 0 & 0
\end{array}\right]\left[\begin{array}{l}
x_{1} \\
x_{2} \\
x_{3} \\
x_{4}
\end{array}\right]}
\end{gathered}
$$

Transfer Function Matrix:

$$
G(s)=C(S I-A)^{-1} B,\left[\begin{array}{l}
Y_{1}(s) \\
Y_{2}(s)
\end{array}\right]=G(s) *\left[\begin{array}{c}
U_{1}(s) \\
U_{2}(s)
\end{array}\right]
$$

$$
\begin{aligned}
& \mathrm{G}(\mathrm{s})= \\
& {\left[\begin{array}{c}
\frac{3 \gamma_{1} k_{1}}{A_{1}\left(S+\left(1 / \tau_{1}\right)\right)} \\
\frac{3\left(1-\gamma_{1}\right) K_{1}}{A_{4} \tau_{2}^{\prime}\left(S+\left(1 / \tau_{2}\right)\right)\left(S+\left(1 / \tau_{4}\right)\right)}
\end{array}\right.}
\end{aligned}
$$

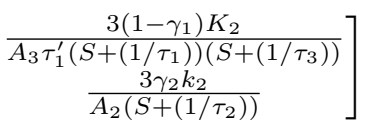

\section{System Analysis}

The rate of change of liquid height $\left(h_{1}, h_{2}, h_{3}, h_{4}\right)$ and relates the difference between inlet flow and outlet flow.

\section{Conical Tank Common Parameter:-}

$\mathrm{H}=$ Tank Height $(60 \mathrm{~cm}) \mathrm{R}$ Outer Radius of conical tank. $=12 \mathrm{~cm}, \mathrm{r}=$ Radius of the outlet $=1 \mathrm{~cm} \mathrm{\&} a_{1}$, $a_{2}, a_{3}$, and $a_{4}=$ cross sectional area of the outlet $=$ $3.14 \times 12=3.14 \mathrm{~cm}^{2}$

Non-minimum Phase QCTS Model found as follows:

$$
G(s)=\left[\begin{array}{cc}
\frac{0.0706}{s+0.488} & 0.017429 \\
0.01641 & \frac{0.070}{(s+0.488)(s+0.8718)} \\
\frac{0.4127)(s+0.62104)}{(s+0.4127)} & \frac{(s+0.412)}{1}
\end{array}\right]
$$

\begin{tabular}{|l|l|}
\hline Parameter & Parameters Value \\
\hline$h_{1}, h_{2}$ & $21.39 \mathrm{~cm}, 18.69 \mathrm{~cm}$ \\
& \\
\hline$h_{3}, h_{4}$ & $14.83 \mathrm{~cm}, 13.48 \mathrm{~cm}$ \\
\hline$\gamma_{1}, \gamma_{2}$ & $0.25,0.30$ \\
\hline$K_{1}, K_{2}$ & $3.3,3.15$ \\
\hline$A_{1}, A_{2}, A_{3}, A_{4}$ & $159.407 \mathrm{~cm}^{2}$ \\
\hline $1 / \tau_{1}, 1 / \tau_{2}$ & $0.39,0.48$ \\
\hline $1 / \tau_{3}, 1 / \tau_{4}$ & $0.93,1.37$ \\
\hline $\mathrm{A}$ & $153.86 \mathrm{~cm}^{2}$ \\
\hline $1 / \tau_{1}{ }^{\prime}, 1 / \tau_{2}{ }^{\prime}$ & $0.47,0.77$ \\
\hline $\mathrm{g}$ & $981 \mathrm{~cm}^{2} / \mathrm{sec}$ \\
\hline
\end{tabular}

Table 1: QCTS parameters for Non-Minimum phase configuration.
QCTS illustrated in Figure 1 where, $h=\left[h_{1}, h_{2}\right]$ is the output vector of QCTS, $h=\left[h_{0}^{1}, h_{0}^{2}\right]^{T}$ is the operating output of $v=\left[v_{1}, v_{2}\right]^{T}$ is control voltage to the pump, the system, $v=\left[v_{0}^{1}, v_{0}^{2}\right]^{T}$ is control voltage at the operating point, $R=\left[r_{1}, r_{2}\right]^{T}$ is reference input.

\subsection{QCTS open loop response for non-minimum phase configuration}

In figure 2, depicted open loop response of QCTS with non-minimum phase configuration

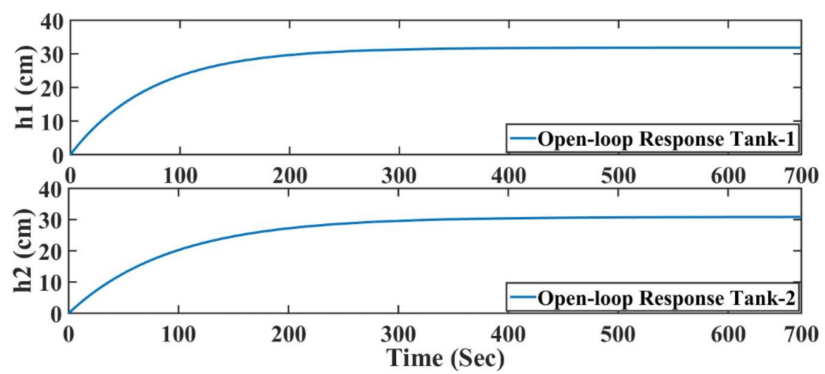

Figure 2: Open-Loop Response of QCTS.

\section{$3 \quad$ FTC Scheme using IT2FLC}

\subsection{Preliminary of Interval type-2 Fuzzy Logic System}

\subsubsection{Type-2 Fuzzy Sets}

The concept of Type-2 Fuzzy Sets was developed by Zadeh already in 1975 as an extension of Type-1 FSs [46] but it only gained broader audience much more recently with the several developments proposed by Mendel and Karnik [16]. Type-1 FSs introduced an important fuzziness degree to create linguistic partitions of a crisp domain. Nonetheless, the MFs used to do so are themselves crisp since they are totally defined without considering any uncertainty on their parameters. Type-2 FS overcome this limitation by defining a secondary degree of fuzziness, i.e. the membership value for each input of a FS is itself defined as a FS in the $[0,1]$ domain [14]. The union of all embedded Type-1 FSs eventually will end up in a blurred area, known as Footprint of Uncertainty (FOU), that is bounded by two MFs, namely the Upper Membership Function (UMF) and the Lower Membership Function (LMF). Furthermore, each membership function given by a person can be assigned with a variable weight according to the amount of confidence associated to its opinion, defining this way the secondary degree of fuzziness. For this reason, a Type-2 FS representation embeds additional degrees of freedom which can better handle uncertainties caused by noisy data and 
changing environments as is required for example when developing a processs model.Type-2 FSs, which can be generically represented by equation (9).

$$
\begin{array}{r}
\tilde{F}=\int_{u \in X} \mu(u) d u=\int_{u \in X} \int_{u \in J_{x}}[g(x) d x] d u \\
J_{x} \subseteq \Re \text { and } X \subset[0,1]
\end{array}
$$

where $g(x)$ is one of the possible primary MFs.

\subsubsection{Type-2 Fuzzy Logic Systems}

The structure of a Type-2 FLS shares the same core components of its Type-1 counterpart, namely: a Fuzzifier, a Rule-Base, an Inference Engine and ultimately the Output Processor [43][44][45]. While in Type-1 FLSs their final stage resumes to a defuzzification procedure, in the Type-2 case the Output Processor embraces an additional stage so a Type- 2 FS is firstly converted into an equivalent Type-1 FS [31]. This procedure is implemented by a Type-Reduction (TR) algorithm, which will be presented further in this document. The interdependency of the referred blocks is depicted in figure (3).

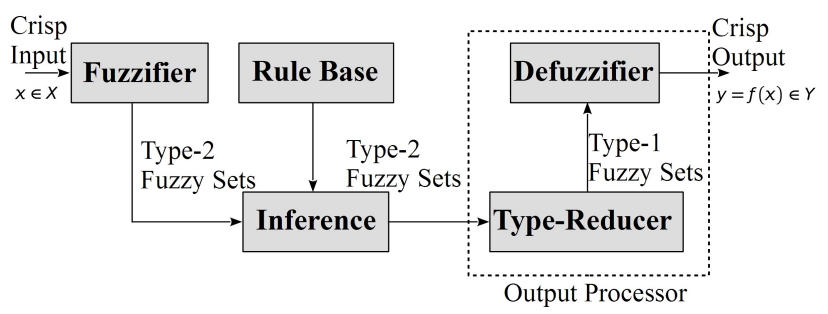

Figure 3: Type-2 Fuzzy Logic System structure [31].

\subsubsection{Fuzzifier}

Similarly to the Type-1 FLS, the most simple way to implement the fuzzifier of a Type-2 FLS is to map a crisp input into a Singleton FS, as defined in equation (10). While information uncertainty is not explicitly considered in the fuzzification stage, it is indirectly accounted for in the rules FSs representations.

$$
\mu_{\tilde{A}_{x}}(x)=\left\{\begin{array}{l}
1, \quad x=x^{\prime} \\
0, \quad \text { otherwise }
\end{array}\right.
$$

where $x^{\prime}$ is the systems input value.

\subsubsection{Rule-Base}

As a natural extension of Type-1 FLS, Type-2 FLSs also synthesize their Rule-Base in a set of If-Then rules, establishing the relations between the systems input and output. Regardless the Fuzzy Sets nature, the way which rules are formed remains the same. Therefore, a Type-2 FLS rule is represented as follows:

$R^{i}: I F x_{1}$ is $F_{1}^{i}$ and $\cdots$ and $x_{j}$ is $F_{j}^{i}$, THEN $y^{i}$ is $\tilde{G}^{i}$

where $R^{i}$ represents the $i^{t h}$ fuzzy rule, $\tilde{F}_{i}^{j}$ j and $\tilde{G}^{i}$ are linguistic terms characterized by Interval Type-2 FSs, $\mathrm{i}=[1, \cdots, \mathrm{M}]$ where $\mathrm{M}$ is the number of rules, $\mathrm{j}=$ $[1, \cdots, \mathrm{N}]$ where $\mathrm{N}$ is the number of antecedents, $x_{j}$ are the FLS inputs and $y_{i}$ is the rule output.

\subsubsection{Inference Engine}

The main difference between a Type-1 FLS and a Type-2 FLS resides in their inference engine. From section 2.2, one concluded that the result of the $j^{\text {th }}$ input and corresponding antecedent operations in the $i^{t h}$ rule yields a crisp number $\left(\mu_{j}^{i}\right)$ referred as membership degree. In an IT2FS the result of this operation is an interval given by $\left(\tilde{\mu}_{j}^{i}\right)$ as follows:

$$
\tilde{\mu}_{F_{j}^{i}}\left(x_{j}\right)=\left[\underline{\mu}_{\tilde{F}_{j}^{i}}\left(x_{j}\right), \bar{\mu}_{\tilde{F}_{j}^{i}}\left(x_{j}\right)\right]
$$

where $x_{j}$ is the $j^{\text {th }}$ FLS system input.

Despite the apparent complexity of this result, an interval based representation allows the direct use of the basic fuzzy logic operations (union, (s-norm), intersection (t-norm) and complement (c-norm)). As so, the t-norm operator, which is used to perform the intersection of the antecedent FS is defined as:

$$
\underline{f}^{i}=T_{j=1}^{N} \underline{\mu}_{\tilde{F}_{i}^{j}}\left(x_{j}\right), \bar{f}^{i}=T_{j=1}^{N} \bar{\mu}_{\tilde{F}_{i}^{j}}\left(x_{j}\right)
$$

where $\mathrm{T}$ is a t-norm (product).

\subsubsection{Type-Reduction}

The Karnik-Mendel (KM) algorithm [42], which can be seen as an extension of Type-1 defuzzification procedure, is currently the most accurate TR method found in literature. Though, given its iterative nature, it is the most complex stage of the fuzzy inference process, requiring extensive calculations even when the simpler IT2FSs are used.

\section{Karnik-Mendel Type-Reduction}

The KM algorithm is an iterative process which allows one to obtain an interval of uncertainty for the centroid of an Interval Type-2 FS given by $\left[y_{l}, y_{r}\right]$. Similarly to the Type-1 FSs defuzzification case, Karnik and Mendel [30] proposed several methods to perform the Type-2 FSs TR based on well known approaches from the Type-1 FLS defuzzification procedures, namely: Height and Modified Height TR, Centroid TR and Center-of-Sets TR. 
Centroid TR the Centroid TR starts by obtaining K samples from a Type-2 FS. Since the FOU of a Type-2 FS embeds several Type-1 FS, to perform the TR one has firstly to obtain two Type-1 FS whose centroid best approximates the upper and lower bounds of the Type2 FS centroid. Using as example the $\tilde{G}_{\text {out }}$ FS, this procedure starts by using its sampled upper and lower bounds to find the optimal values for the switching points $[\mathrm{L}, \mathrm{R}]$, as depicted in figure (2.17).

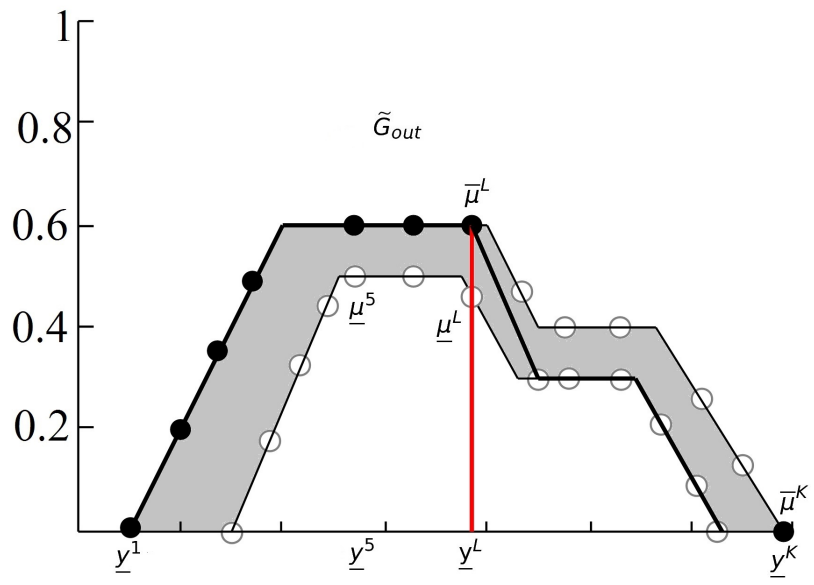

Figure 4: Computing $y_{l}$ : Switching from the upper bounds of the firing intervals to the lower bounds.

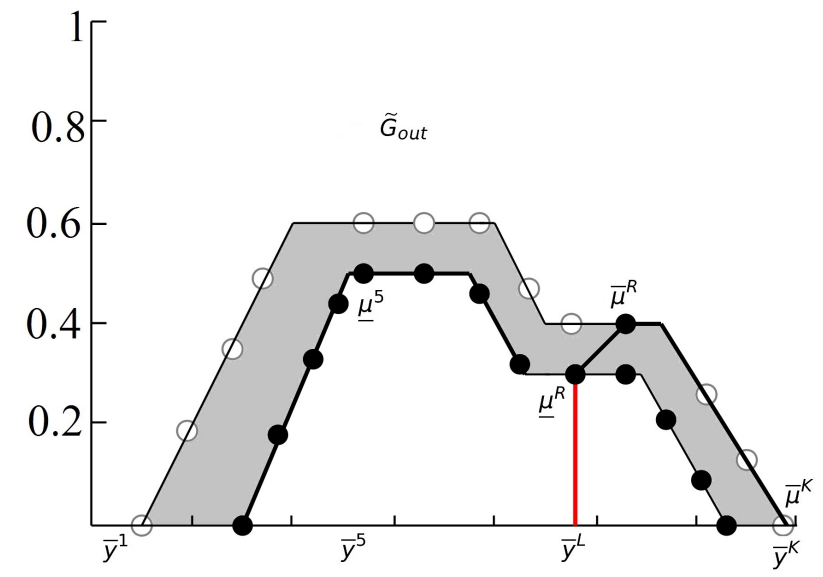

Figure 5: Computing $y_{r}$ : Switching from the lower bounds of the firing intervals to the upper bounds.

The candidate points are obtained as follows in equations (14) and (15).

$$
y_{l}(k)=\frac{\sum_{i=1}^{k} \underline{y}^{i} \bar{\mu}_{\tilde{G}_{o}}^{i}+\sum_{i=k+1}^{K} \underline{y}^{i} \underline{\mu}_{\tilde{G}_{o}}^{i}}{\sum_{i=1}^{k} \bar{\mu}_{\tilde{G}_{o}}^{i}+\sum_{i=k+1}^{K} \underline{\mu}_{\tilde{G}_{o}}^{i}}
$$

$$
y_{r}(k)=\frac{\sum_{i=1}^{k} \bar{y}^{i} \underline{\mu}_{\tilde{G}_{o}}^{i}+\sum_{i=k+1}^{K} \bar{y}^{i} \bar{\mu}_{\tilde{G}_{o}}^{i}}{\sum_{i=1}^{k} \underline{\mu}_{\tilde{G}_{o}}^{i}+\sum_{i=k+1}^{K} \bar{\mu}_{\tilde{G}_{o}}^{i}}
$$

where $\mathrm{k}$ is an integer in $[1, \mathrm{~K}-1]$ interval, where $\mathrm{K}$ is the number of discretization points. Then, the optimal interval bounds can be obtained by $y_{l}$ and $y_{r}$, as following presented:

$$
\begin{gathered}
y_{l}=\min _{k \in[1, M-1]} y_{l}(k) \equiv y(L) \equiv \\
\frac{\sum_{i=1}^{L} \underline{y^{i}} \bar{\mu}_{\tilde{G}_{o}}^{i} \sum_{i=L+1}^{K} \underline{y^{i}} \underline{\mu}_{\tilde{G}_{o}}^{i}}{\sum_{i=1}^{L} \bar{\mu}_{\tilde{G}_{o}}^{i}+\sum_{i=L+1}^{K} \underline{\mu}_{\tilde{G}_{o}}^{i}} \\
y_{r}=\max _{k \in[1, M-1]} y_{r}(k) \equiv y(R) \equiv \\
\frac{\sum_{i=1}^{R} \bar{y}^{i} \underline{\mu}_{\tilde{G}_{o}}^{i} \sum_{i=R+1}^{K} \bar{y}^{i} \bar{\mu}_{\tilde{G}_{o}}^{i}}{\sum_{i=1}^{R} \underline{\mu}_{\tilde{G}_{o}}^{i}+\sum_{i=R+1}^{K} \bar{\mu}_{\tilde{G}_{o}}^{i}}
\end{gathered}
$$

where $\mathrm{L}$ and $\mathrm{R}$ are switch points satisfying

$$
\begin{aligned}
y^{L} \leq y_{l}<y^{L+1} \\
y^{R} \leq y_{r}<y^{R+1}
\end{aligned}
$$

\subsubsection{Defuzzifier}

After applying one of the possible TR methods, the obtained Interval Fuzzy Set still has to be converted into a crisp number so it becomes suited to the most part of the FLS application scenarios. Anyway, this procedure is fairly straightforward, and the defuzzified value obtained by simply computing the average of the intervals left and right endpoints.

$$
y_{\text {out }}=\frac{y_{r}+y_{l}}{2}
$$

\subsubsection{Membership function}

For designing the proposed controller IT2FLC we used Gaussian membership function is used which defined by equation (20).

$$
\begin{array}{r}
\tilde{F}_{i}\left(c_{i}, \sigma_{i}, x\right)=\exp \left[-\frac{1}{2}\left(\frac{x-c_{i}}{\sigma_{i}}\right)^{2}\right]= \\
G\left(c_{i}, \sigma_{i}, x\right), \sigma_{i}^{j} \in\left[\sigma 1_{i}, \sigma 2_{i}\right] \text { and } c_{i} \in\left[c 1_{i}, c 2_{i}\right]
\end{array}
$$

\subsection{Design of IT2FLC}

Fault-tolerant control structure design proposed using IT2FLC for QCTS with two possible faults in abrupt and incipient nature.The proposed FTC scheme presented in figure 6. As depicted in FTC structure in figure 6, proposed FTC using IT2FLC is model base approach and for detecting the faults residue signal $(r)$ 
is there and two other signal is generated one is error (e) and second one is rate of Chang of error $\dot{e}$. One controller output is there which controlling the QCTS output vector $\left[h_{1}, h_{2}\right]$

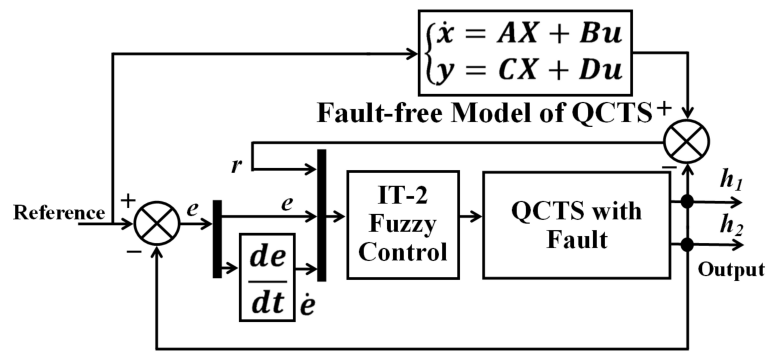

Figure 6: Proposed FTC Scheme using IT2 FLC.
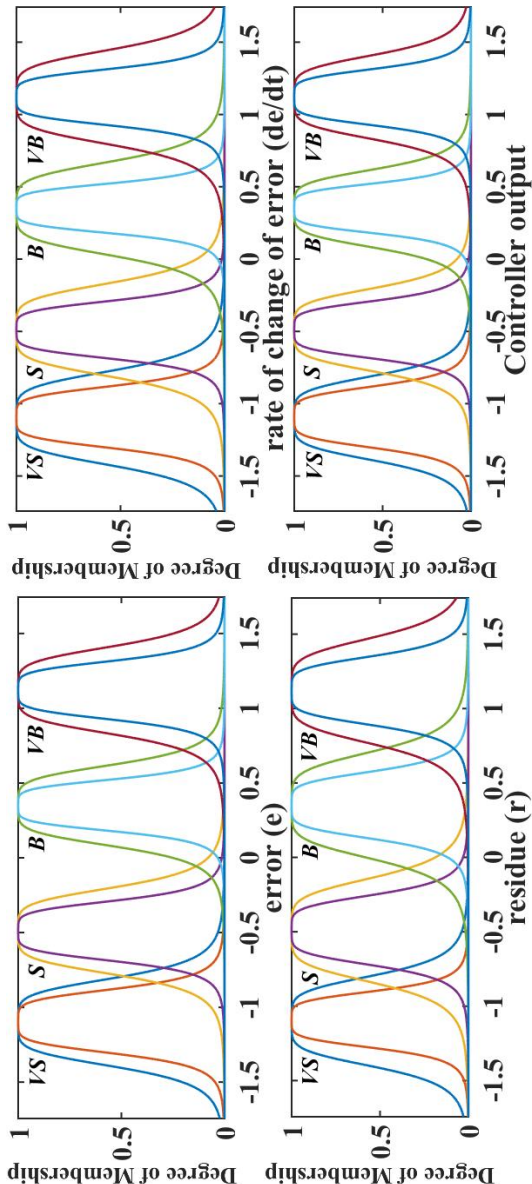

Figure 7: Membership Function for IT2FLC.

For designing IT2FLC three input and one output is taken. Each input and output having four linguistic variable,namely VS: Very small, S: small, B: big, and VB: very big.proposed FLC design with gaussian membership function the, equation (20) represents the mathematical representation of that function.

The highest possibility of rule- no.= input $1 \mathrm{MF}$ no. of linguistic variables $\times$ input $2 \mathrm{MF}$ no. of linguistic variables $\times$ input $3 \mathrm{MF}$ no. of linguistic variables

We design the 64 possible rule-base for proposed IT2FLC.

The membership function range is design by prepossessing of the input and output values and found is [-1.7431.743]. The input and output membership function of IT2FLC is presents in figure 7.

\section{Simulation Results}

The simulation results of proposed scheme is compared with other conventional and proven strategy like $\mathcal{H}_{\infty}$, LQR, 2 DoF Decentralized PI Controller and model free Type 1 Fuzzy Logic controller (T1FLC). The LQR and $\mathcal{H}_{\infty}$ design for Quadruple Tank System (QTS) is proposed in [32], the same strategy is applied on Quadruple Conical Tank System (QCTS) subject to two possible faults ( actuator fault $\left(f_{a}\right)$ and system component fault $\left.\left(f_{\text {sys }}\right)\right)$ in two different nature abrupt and incipient in nature respectively.

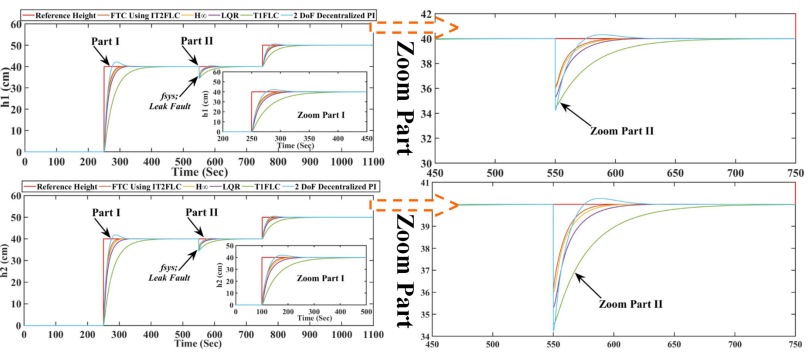

Figure 8: Regulatory responses of QCTS with (abrupt) system component faults $\left(f_{\text {sys }}\right)$.

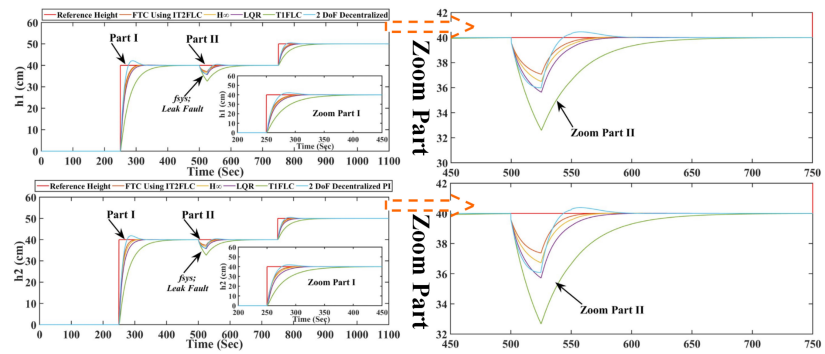

Figure 9: Regulatory responses of QCTS with (incipient) system component faults $\left(f_{\text {sys }}\right)$.

Abrupt and incipient nature of system component fault $f_{\text {sys }}$ is introduce on QCTS, and simulate the proposed FTC with others strategy. The simulation results presented in figure (8) \& figure (9).Validation of the simulation results is demonstrated using three 
integral error which is clearly shows that proposed controller IT2FLC is giving superior response when system component fault accrue in the system. The comparative error results illustrated in table 2 and table 3 . Table 1 presents the transient response for the Abrupt
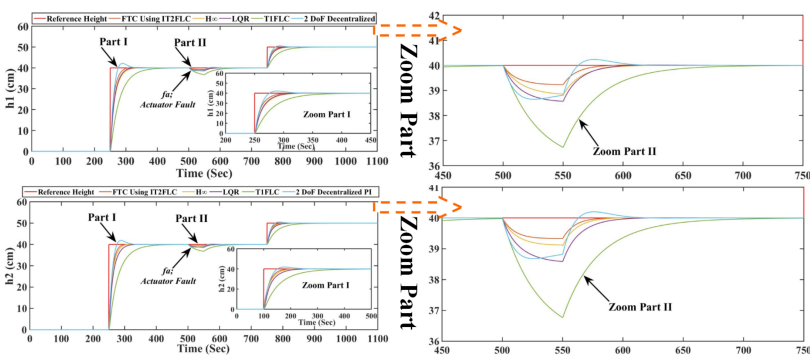

Figure 10: Regulatory responses of QCTS with (abrupt) actuator faults $\left(f_{a}\right)$.

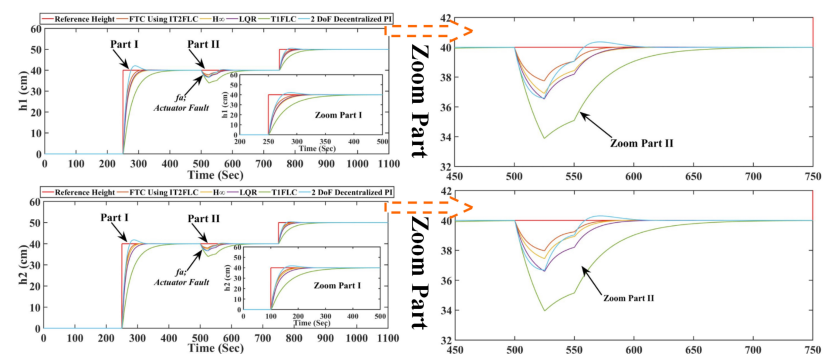

Figure 11: Regulatory responses of QCTS with (incipient) actuator faults $\left(f_{a}\right)$.

and incipient nature of system component fault $f_{a}$ is introduce on QCTS, and simulate the proposed FTC with others strategy. The simulation results presented in figure (10) \& figure (11). simulation results clearly shows that proposed controller IT2FLC is giving superior response when actuator fault accrue in the system. The comparative error results illustrated in table 4 and table 5.

\begin{tabular}{|l|l|l|l|}
\hline \multicolumn{5}{|c|}{ Transient Response } \\
\hline $\begin{array}{l}\text { Controller Used in } \\
\text { FTC }\end{array}$ & $\begin{array}{l}M_{p} \\
(\mathrm{Sec})\end{array}$ & $\begin{array}{l}T_{s} \\
(\mathrm{Sec})\end{array}$ & $\begin{array}{l}T_{r} \\
(\mathrm{Sec})\end{array}$ \\
\hline IT2FLC & 0 & 57 & 17 \\
\hline$H_{\infty}$ & 0 & 74 & 34 \\
\hline LQR & 0 & 76 & 36 \\
\hline T1FLC & 0 & 155 & 70 \\
\hline $\begin{array}{l}\text { 2 DoF Decentralized } \\
\text { PI }\end{array}$ & 2.54 & 128 & 38 \\
\hline
\end{tabular}

Table 2: Transient response specification compassion.

\begin{tabular}{|l|c|c|c|c|}
\hline \multicolumn{4}{|c|}{ Integral Error Compression } \\
\hline $\begin{array}{l}\text { Controller Used in } \\
\text { FTC }\end{array}$ & IAE & ISE & ITAE \\
\hline IT2FLC & 36.94 & 54.74 & 96.11 \\
\hline$H_{\infty}$ & 46.84 & 63.99 & 104.92 \\
\hline LQR & 49.98 & 74.83 & 114.92 \\
\hline T1FLC & 56.38 & 79.19 & 121.14 \\
\hline $\begin{array}{l}\mathbf{2} \text { DoF Decentralized } \\
\text { PI }\end{array}$ & 61.84 & 92.91 & 146.11 \\
\hline
\end{tabular}

Table 3: Integral error compression for regulatory response of QCTS with abrupt nature of system component faults $\left(f_{\text {sys }}\right)$.

\begin{tabular}{|l|c|c|c|}
\hline \multicolumn{4}{|c|}{ Integral Error Compression } \\
\hline $\begin{array}{l}\text { Controller Used in } \\
\text { FTC }\end{array}$ & IAE & ISE & ITAE \\
\hline IT2FLC & 32.67 & 48.24 & 89.81 \\
\hline$H_{\infty}$ & 39.91 & 58.25 & 98.12 \\
\hline LQR & 41.69 & 68.91 & 111.93 \\
\hline T1FLC & 43.3 & 74.93 & 119.82 \\
\hline $\begin{array}{l}\mathbf{2} \text { DoF Decentralized } \\
\text { PI }\end{array}$ & 51.47 & 88.69 & 134.21 \\
\hline
\end{tabular}

Table 4: Integral error compression for regulatory response of QCTS with incipient nature of system component faults $\left(f_{\text {sys }}\right)$.

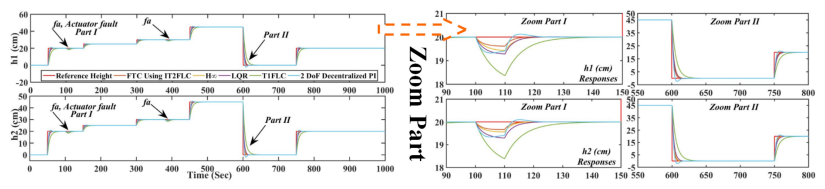

Figure 12: Servo responses of QCTS with system component faults (abrupt).

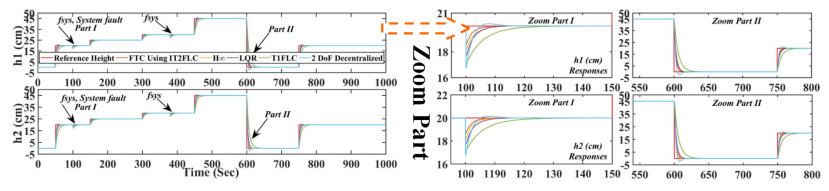

Figure 13: Servo responses of QCTS with (abrupt) actuator faults $\left(f_{a}\right)$.

Also, proposed IT2FLC and other controllers are verified with servo response on QCTS with (abrupt) actuator $\left(f_{a}\right)$ and system component $\left(f_{\text {sys }}\right)$ faults. In figure (12) \& figure (13) comparative responses of QCTS is 


\begin{tabular}{|l|c|c|c|}
\hline \multicolumn{4}{|c|}{ Integral Error Compression } \\
\hline $\begin{array}{l}\text { Controller Used in } \\
\text { FTC }\end{array}$ & IAE & ISE & ITAE \\
\hline IT2FLC & 38.96 & 56.01 & 99.15 \\
\hline$H_{\infty}$ & 49.75 & 66.82 & 108.45 \\
\hline LQR & 52.74 & 76.62 & 118.62 \\
\hline T1FLC & 55.31 & 81.73 & 125.12 \\
\hline $\begin{array}{l}\mathbf{2} \text { DoF Decentralized } \\
\text { PI }\end{array}$ & 68.87 & 96.84 & 152.82 \\
\hline
\end{tabular}

Table 5: Integral error compression for regulatory response of QCTS with abrupt nature of actuator faults $\left(f_{a}\right)$.

\begin{tabular}{|l|c|c|c|}
\hline \multicolumn{4}{|c|}{ Integral Error Compression } \\
\hline $\begin{array}{l}\text { Controller Used in } \\
\text { FTC }\end{array}$ & IAE & ISE & ITAE \\
\hline IT2FLC & 33.63 & 49.81 & 88.48 \\
\hline$H_{\infty}$ & 39.23 & 59.34 & 99.82 \\
\hline LQR & 42.24 & 71.87 & 112.95 \\
\hline T1FLC & 44.81 & 74.83 & 120.86 \\
\hline $\begin{array}{l}\mathbf{2} \text { DoF Decentralized } \\
\text { PI }\end{array}$ & 52.52 & 89.84 & 137.82 \\
\hline
\end{tabular}

Table 6: Integral error compression for regulatory response of QCTS with incipient nature of actuator faults $\left(f_{a}\right)$.

presented, which clearly shows that proposed IT2FLC giving zero steady-state error, smooth reference tracking trajectory, and zero overshot as compared to other controllers.

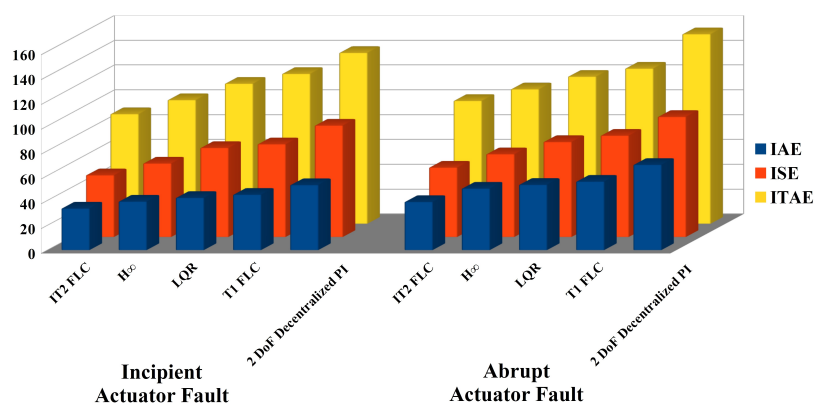

Figure 14: The integral error variations for different controllers for QCTS process subject to system component faults $\left(f_{\text {sys }}\right)$.

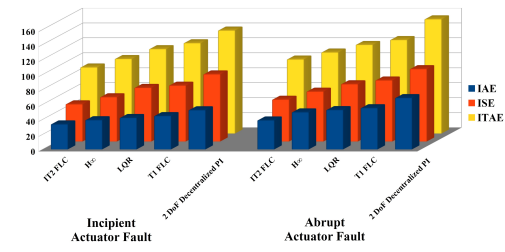

Figure 15: The integral error variations for different controllers for QCTS process subject to actuator faults $\left(f_{a}\right)$.

\section{Conclusion}

This paper presented simple and effective FTC scheme using interval type-2 fuzzy logic (IT2FLC) for highly nonlinear (QCTS) process in non-minimum phase configuration. Out of three possible faults in QCTS article considering two possible faults one is system component $\left(f_{\text {sys }}\right)$ fault and other one is actuator $\left(f_{a}\right)$ fault. Also, the two different fault characteristic is taken (i.e. abrupt and incipient).Simulation results are validated through integral error indices. The simulation results prove that the proposed IT2FLC scheme outperform the $\mathcal{H}_{\infty}$, LQR, 2 DoF Decentralized PI Controller and model free Type 1 Fuzzy Logic controller (T1FLC) solution previously proposed, in terms of regulatory closed loop response, servo performance, and fault tolerance under nominal conditions. The settling time, rise time and the overshoot obtained and compared with previously proposed controller. The most important contribution of the IT2FLC scheme is the fast response with zero overshot, compared to the other given approach. Further research includes the linearized model of QCTS in MATLAB platform for nonminimum phase configuration, and open-loop response is carried out from that model.

Finally, IT2FLC is proposed and simulated on QCTS subject to two faults. The experimental validation is not covered in this article, this is a subject of future research and can be considered as natural extension of this work. However the computation burden is one of the key limitation to implementation of IT2 FLC. To overcome this limitation number of algorithms are implemented, one of the Cuckoo Search algorithm is discussed in [36] for optimized Takagi-Sugeno-Kang Consequents.

As future work we will consider evaluating the FLC by variating the MFs of the input-outputs fuzzy sets and optimized the MFs as some in [13] literature discussed related to this and give optimum performance. In addition, include Comparative analysis of noise robustness of type 2 fuzzy logic controllers. 


\section{Acknowledgement}

The authors are grateful to the Department of Instrumentation and Control Engineering, Nirma University, Ahmedabad India, for providing data related to QCTS prototype system to identify the mathematical model.The authors would like to thank the Dr.Jignesh B. Patel, Sr.Associate Professor, Department of Instrumentation and Control Engineering, Nirma University, Ahmedabad, India, We also like to thank the anonymous referee for strong technical suggestions which are helping us to improve the quality of the paper.

\section{References}

[1] P. J. P. R. A. Patel, P. Joshi, J. Chirag, Parametric analysis of quadruple conical tank system, in: Nirma University International Conference on Engineering (NUiCONE), IEEE, Ahmedabad, India, 23-25 Nov. 2017, pp. 1-6.

[2] M. B. Cosenza, Experimental comparison of type1 and type-2 fuzzy logic controllers for the control of level and temperature in a vessel, Computer Aided Chemical Engineering 29 (2011) 803-807.

[3] W. Belhaj, O. Boubaker, On mimo pid control of the quadruple-tank process via ilmis approaches: minimum and non-minimum case studies dynamics and control of process systems, in: In: IFAC Proceedings International symposium on dynamic and control of process systems, IFACPapers Online, Mumbai, India, 1820 December 2013.

[4] W. Bequette, Process Control: Modeling, Design and Simulation, Prentice Hall Professional, New Jersey, 2003.

[5] B. L. C. Rimpy, D. Himadri, K. Rajesh, Comparative analysis of quadruple conical tank and cylindrical tank system., International Journal of Scientific \& Engineering Research, 5 (2014) 16081616.

[6] O. Castillo, L. Amador-Angulo, J. R. Castro, M. G. Valdez, A comparative study of type-1 fuzzy logic systems, interval type-2 fuzzy logic systems and generalized type-2 fuzzy logic systems in control problems, Inf. Sci. 354 (2016) $257-$ 274 .

[7] L. Cervantes, O. Castillo, Type-2 fuzzy logic aggregation of multiple fuzzy controllers for airplane flight control, Information Sciences 324 (C) (2015) 247-256.

URL https : //doi.org/10.1016/j .ins.2015.06.047
[8] B. M. E. Govinda Kumar, N. Dhivya, Enhancement of pid controller performance for a quadruple tank process with minimum and nonminimum phase behaviors, Procedia Technology (2014) 480-489.

[9] O. C. E. Ontiveros-Robles, P. Melin, Comparative analysis of noise robustness of type 2 fuzzy logic controllers, Kybernetika 54(1) (2018) 175-201.

[10] R. J. M. F. Garelli, H. D. Battista, Limiting interactions in decentralized control of mimo system, Journal of Process Control 16 (2006) 473-483.

[11] R. J. M. F. Garelli, H. D. Battista, Partial decoupling of non-minimum phase processes with bounds on the remaining coupling., Chemical Engineering Science 61 (2006) 7706-7716.

[12] H. Hagras, Type-2 flcs: A new generation of fuzzy controllers, IEEE Computational Intelligence Magazine 2 (1) (2007) 30-43.

[13] P. M. F. V. L. Cervantes, O. Castillo, Genetic optimization of membership functions in modular fuzzy controllers for complex problems, in: J. K. O. Castillo, P. Melin (Ed.), Recent Advances on Hybrid Intelligent Systems. Studies in Computational Intelligence, Vol. 451, Springer Heidelberg, Berlin, 2013, pp. 51-62.

[14] J. Mendel, Uncertain Rule-Based Fuzzy Logic Systems: Introduction and new directions, Springer International Publishing, 2001.

[15] E. Mirakhorli, M. Farrokhi, Sliding-mode statefeedback control of non-minimum phase quadruple tank system using fuzzy logic, Proceedings of the 18th IFAC World Congress 1 (2011) 1354613551 .

[16] J. M. N. Karnik, Q. Liang, Type-2 fuzzy logic systems, IEEE Transactions on Fuzzy Systems 7 (6) (1999) 643-658.

[17] J. S. M. S. O. Castillo, L.Cervantes, J. R.Castro, A generalized type-2 fuzzy granular approach with applications to aerospace, Information Sciences 354(1) (2016) 165-177.

[18] H. R. Patel, V. A. Shah, Fault detection and diagnosis methods in power generation plants - the indian power generation sector perspective: An introductory review, Journal of Energy and Management 2(2) (2018) 31-49.

[19] H. R. Patel, V. A. Shah, A fault-tolerant control strategy for non-linear system: An application to the two tank canonical noninteracting level control system, in: in Proc. IEEE Distributed Computing, VLSI, Electrical Circuits 
and Robotics (DISCOVER-2018), IEEE, Mangalore, India, 2018, pp. 64-70.

[20] H. R. Patel, V. A. Shah, Fault tolerant control systems: A passive approaches for single tank level control system, i-managers Journal on Instrumentation and Control Engineering 6(1) (2018) 11-18.

[21] H. R. Patel, V. A. Shah, A framework for fault-tolerant control for an interacting and noninteracting level control system using ai, InProceedings of the 15th International Conference on Informatics in Control, Automation and Robotics I (2018) 180-190.

[22] H. R. Patel, V. A. Shah, Fuzzy logic based passive fault tolerant control strategy for a singletank system with system fault and process disturbances, in: in Proc. 5th International Conference on Electrical and Electronics Engineering (ICEEE-2018), IEEE, Istanbul, Turkey, 2018, pp. $257-262$.

[23] H. R. Patel, V. A. Shah, Passive fault-tolerant control system design with tracking control against major system faults: Application to a canonical tank level system, in: 2nd International Conference on Communication and Computing Systems, Dronacharya College of Engineering, (ICCCS-2018), Taylor \& Francis To be Published, Gurgaon, India,, 2018.

[24] H. R. Patel, V. A. Shah, Actuator and system component fault tolerant control using interval type-2 takagi-sugeno fuzzy controller for hybrid nonlinear process, International Journal of Hybrid Intelligent Systems. Pre-press (2019) 1-11.

[25] H. R. Patel, V. A. Shah, Decentralized fault tolerant fuzzy plus pi control of mimo systems: Quadruple tank case study, in: in Proc. The International Conference on Innovative Applied Energy (IAPE'19), Oxford, United Kingdom, 2019, pp. $1-11$.

[26] H. R. Patel, V. A. Shah, Fault tolerant controller using interval type-2 tsk logic control systems: Application to three interconnected conical tank system, 38th North American Fuzzy Information Processing Society Annual Conference. (to be published).

[27] H. R. Patel, V. A. Shah, Passive fault tolerant control system for two-tank interacting conical level control system against partial actuator failures, Journal of Computer Science. (to be published).
[28] H. R. Patel, V. A. Shah, Passive fault tolerant controller using hybrid controller for effective control of two tank canonical non-interacting system, Journal of Mathematics and Statistics. (to be published).

[29] H. R. Patel, V. A. Shah, Performance comparison of passive fault tolerant control strategy with pi and fuzzy control of single-tank level process with sensor and system fault, American Journal of Engineering and Applied Sciences. (to be published).

[30] H. R. Patel, V. A. Shah, Shah.,a passive faulttolerant control strategy for non-linear system: An application to the two tank conical noninteracting level control system, MASKAY 9(1) (2019) 1-8.

[31] H. R. Patel, V. A. Shah, Fault tolerant control using interval type-2 takagi-sugeno fuzzy controller for nonlinear system, in: P. M. N. G. A. Abraham, A. Cherukuri (Ed.), Intelligent Systems Design and Applications, Vol. 941, Springer, Cham, Switzerland, 2020, pp. 150-164.

[32] J. K. Pradhan, A. Ghosh, Multi-input and multioutput proportional-integral-derivative controller design via linear quadratic regulator-linear matrix inequality approach., IET Control Theory 9 (2015) 2140-2145.

[33] E. P. G. R. Vadigepalli, F. J. D. III, Robust control of a multivariable experimental four-tank system., International Journal of Computer Applications 40 (2001) 1916-1927.

[34] N. S. S. K. Lakshmanaprabu, U. S. Banu, Closed loop control of quadruple tank process using fuzzy logic pi controller., International Journal of Computer Applications CiQs(1) (2014) 20-24.

[35] M. A. Sanchez, O. Castillo, J. R. Castro, Generalized type-2 fuzzy systems for controlling a mobile robot and a performance comparison with interval type-2 and type-1 fuzzy systems, Expert Systems with Applications 42 (14) (2015) 5904-5914.

URL http://dx.doi.org/10.1016/j . eswa. 2015.03.02

[36] M. A. Sanchez, O. Castillo, J. R. Castro, Information granule formation via the concept of uncertainty-based information with interval type-2 fuzzy sets representation and takagisugeno-kang consequents optimized with cuckoo search, Applied Soft Computing 27 (C) (2015) 602-609.

URL http://dx.doi.org/10.1016/j .asoc. 2014.05.03 
[37] J. M. K. M. Soroushb, W. D. Seidera, Nonlinear feedback control of multivariable non-minimumphase processes., Journal of Process Control 12 (2002) 667-686.

[38] M. Suja, T. Thyagarajan, Design of decentralized fuzzy controllers for quadruple tank process, International Journal of Computer Science and Network Security 8 (2008) 163-168.

[39] D. A. Vijula, N. Devarajan, Design of decentralized pi controller using model reference adaptive control for quadruple tank process, International Journal of Engineering and Technology 5 (2014) 5057-5066.

[40] D. A. R. Wati, Maximum power point tracking of photovoltaic systems using simple interval type-2 fuzzy logic controller based on hill climbing algorithm, in: Proc. 2016 Inter- national Seminar on Intelligent Technology and Its Applications (ISITIA), Lombok, Indonesia, 2016, pp. 687-692.

[41] $\mathrm{D} . \mathrm{Wu}, \mathrm{On}$ the fundamental differences between interval type-2 and type-1 fuzzy logic controllers, IEEE Transactions on Fuzzy Systems 20 (5) (2012) 832-848.

[42] D. Wu, W. Tan, Computationally efficient typereduction strategies for a type-2 fuzzy logic controller, in: Proc. 14th IEEE International Conference in Fuzzy Systems (FUZZ '05), Prague, Czech Republic, 2005, pp. 353-358.

[43] L. A. Zadeh, Fuzzy sets, Information and Control 8 (1965) 338-353.

[44] L. A. Zadeh, The concept of a linguistic variable and its application to approximate reasoning I, Information Sciences 8 (1975) 199-250.

[45] L. A. Zadeh, The concept of a linguistic variable and its application to approximate reasoning II, Information Sciences 8 (1975) 301-357.

[46] L. A. Zadeh, The concept of a linguistic variable and its application to approximate reasoning III, Information Sciences 9 (1975) 43-80. 